See discussions, stats, and author profiles for this publication at: https://www.researchgate.net/publication/299058909

\title{
Estudio Piloto del Programa de Entrenamiento en Mindfulness Basado en Prácticas Breves Integradas (M-PBI)
}

Article in Revista de Psicoterapia · March 2016

DOI: 10.33898/rdp.v27i103.110

\section{CITATIONS}

5 authors, including:

Luis Botella

Universitat Ramon Llull

316 PUBLICATIONS 1,303 CITATIONS

SEE PROFILE

Montse Sabate

Trial Form Support International AB

27 PUBLICATIONS 806 CITATIONS

SEE PROFILE

Some of the authors of this publication are also working on these related projects:

Health Promotion: The Impact of Pyschological Factors on Lifestyle View project

Early intervention schizophrenia View project
Marcial Arredondo Rosas

Universitat Ramon Llull

8 PUBLICATIONS 19 CITATIONS

SEE PROFILE 


\section{ESTUDIO PILOTO DEL PROGRAMA DE}

ENTRENAMIENTO EN MINDFULNESS BASADO EN

\section{PRÁCTICAS BREVES INTEGRADAS (M-PBI)}

\section{A PILOT STUDY OF THE MINDFULNESS TRAINING PROGRAM BASED ON BRIEF INTEGRATED PRACTICES (M-BIP)}

\section{Marcial Arredondo Rosas}

Departament de Psicologia Bàsica, Evolutiva i de l'Educació.

Universitat Autònoma de Barcelona.

Mindfulness y Psicología Barcelona.

\section{Montserrat Sabaté Pina}

TFS Develop, Barcelona.

\section{Luis Botella García del Cid}

Facultat de Psicologia, Ciències de l'Educació i l'Esport (FPCEE) Blanquerna.

Universitat Ramon Llull, Barcelona.

\section{Pilar Hurtado Villar}

Institut Mensalus, Barcelona.

Mindfulness y Psicología Barcelona.

\section{Lesly M. Acosta Argueta}

Universitat Politècnica de Catalunya/BARCELONATECH.

Cómo referenciar este artículo/How to reference this article:

Arredondo, M., Sabaté, M., Botella L., Acosta, L.M. y Hurtado, P. (2016). Estudio Piloto del Programa de Entrenamiento en Mindfulness Basado en Prácticas Breves Integradas (M-PBI). Revista de Psicoterapia, 27(103), 151-168. 


\title{
Resumen
}

El objetivo de este estudio es valorar los primeros resultados del programa de mindfulness basado en prácticas breves integradas (M-PBI) en cuanto a su efecto sobre la capacidad de mindfulness (mediante el Five Facet Mindfulness Questionnaire, $F F M Q$ ), descentramiento (Experiences Questionnaire-Decentering, EQ-D) y sintomas de depresión, ansiedad y estrés (Depression Anxiety and Stress Scale, DASS-21). Se incluyeron 74 participantes y la totalidad finalizaron el estudio. La media de edad fue de 38.9 años $(D E=9)$, el 74.3\% fueron mujeres y el 87\% estaban laboralmente en activo. Las puntuaciones medias del FFMQ y EQ-D aumentaron significativamente y las del DASS-21 disminuyeron de forma también significativa en la medición postentrenamiento vs. pre-entrenamiento ( $p<.0001$, en todos los casos). Los cambios más altos se dieron en el aumento de la capacidad de descentramiento (cambio medio de 7.6 puntos; IC95\%: 5.8 a 9.4), disminución del estrés (-6.6; IC95\%: -8.5 a -4.8) y en la mejoría de tres facetas del FFMQ (no juicio, no reactividad y capacidad de observación: 5.8 [IC95\%: 4 a 8.1], 4.9 [IC95\%: 3.5 a 6.5 ] y 5.3 [IC95\%: 4.4 a 6.3 ] respectivamente). Estos cambios parecen no depender de la edad, sexo, estar en tratamiento psicológico, profesión o tener práctica de meditación y motivación para la realización del programa. Como conclusión, estos primeros resultados sugieren que el entrenamiento en el programa M-PBI conlleva un aumento de las capacidades de mindfulness y descentramiento y una reducción de los sintomas de estrés, ansiedad y depresión. No obstante se requieren futuros estudios con un diseño aleatorizado y grupo control para confirmar estos resultados y controlar los posibles factores de confusión, así como minimizar los sesgos.

Palabras clave: mindfulness, $M-P B I$, prácticas breves, FFMQ, DASS-21, EQ-D.

\begin{abstract}
The aim of this study is to assess the first results of a new mindfulness program based on integrated brief practices (M-PBI) regarding its effect on the mindfulness ability (assessed by the Five Facet Mindfulness Questionnaire, FFMQ), decentering (by the Experiences Questionnaire-Decentering, EQ-D), and depression, anxiety, and stress (by the Depression Anxiety and Stress Scale, DASS-21). Seventy-four participants were included and all of them completed the study. The mean (SD) age was 38.9 (9) years, $74.3 \%$ were female, and $87 \%$ employed workers. The mean FFMQ and EQ-D scores increased significantly and the mean DASS-21 scores decreased significantly in the post-training vs. pre-training measurements ( $p<.0001$, in all cases). The highest changes were observed in the decentering ability (mean change of 7.6 points; $95 \% \mathrm{CI}$ : 5.8 to 9.4), decreasing the stress 6.6; 95\% CI: -8.5 to -4.8 points), and in increasing three FFMQ scales (observe, non-judge and non-reactivity: 5.8 [95\% CI: 4 to 8.1], 4.9 [95\% CI: 3.5 to 6.5$]$ y 5.3 [95\% CI: 4.4 to 6.37 respectively). These changes seem to be not associated to age, sex, psychological treatment, profession, previous practice of meditation and motivation to carry out the program. In conclusion, these results show that the M-PBI entails an improvement of the ability of mindfulness and decentering, reduced stress, anxiety and depression. However, future studies with a randomized and control group design are needed in order to confirm these results and to control possible confounding factors and minimize bias.
\end{abstract}

Keywords: mindfulness, M-PBI, brief practices, FFMQ, DASS-21, EQ-D. 
Mindfulness puede definirse como «prestar atención de forma intencionada al momento presente sin juzgar» (Kabat-Zinn, 1994). Simón (2007) lo define como una capacidad humana universal y básica, que consiste en la posibilidad de ser conscientes de los contenidos de la mente momento a momento. La atención plena nos permite darnos cuenta de la actividad mental que se está experimentando, reduciendo la divagación de la mente y manteniendo el foco de la atención durante más tiempo en el momento presente, puesto que habitualmente la mente se encuentra vagando sin orientación, saltando de unas imágenes a otras y de unos pensamientos a otros.

Uno de los programas de mindfulness más utilizados hasta la actualidad para la reducción del estrés es el MBSR (Mindfulness Based Stress Reduction). Este programa fue creado en el año 1979 por el Dr. Jon Kabat-Zinn en el Centro Médico de la Universidad de Massachusetts, y desde entonces se ha utilizado como parte del tratamiento en una gran variedad de problemas de salud, principalmente en pacientes con dolor crónico, cáncer o factores de riesgo de enfermedades coronariasvéase una revisión en Pérez y Botella (2006). Se trata de una intervención psicoeducativa que tiene como finalidad reducir el malestar psicológico y que enfatiza la capacidad de parar y ver antes de actuar, para aprender a desplegar comportamientos más eficaces y adaptados a los acontecimientos. El programa se desarrolla en ocho sesiones semanales de dos horas y media y un día de prácticas intensivas de ocho horas, realizándose prácticas diarias de meditación de 45 minutos a lo largo de todo el programa (Instituto esMindfulness; Bishop, 2002).

Los programas de entrenamiento en mindfulness, como el MBSR, requieren prácticas de meditación de larga duración, lo que dificulta el cumplimiento de muchos de los participantes (Shapiro, Astin, Bishop, \& Cordova, 2005). No obstante, cada vez son más los estudios que tratan de demostrar los beneficios de las prácticas breves de mindfulness, para así hacer llegar la práctica de la meditación a un público mucho más amplio. En este sentido, Fortney, Luchterhand, Zakletskaia, Zgierska y Rakel (2013) desarrollaron una versión abreviada del programa MBSR adaptada a médicos de atención primaria. Este programa incluía prácticas de meditación diarias de 10-20 minutos. Tras la implementación de esta intervención abreviada se observó una mejoría de la satisfacción en el trabajo, la calidad de vida y de la compasión. Zeidan, Johnson, Diamond, David y Goolkasian (2010) demostraron que la implementación de un programa de mindfulness basado en prácticas de mediación breves (20 minutos) durante tres días consecutivos conseguía reducir la tasa subjetiva de dolor percibido, la sensibilidad al dolor y el estado de ansiedad.

El programa de mindfulness que se presenta en este estudio es el M-PBI (Mindfulness basado en Prácticas Breves Integradas). Este programa recoge e integra los aspectos transversales de los programas y postulados de mindfulness que cuentan con evidencia científica. El programa incorpora prácticas breves para facilitar su práctica diaria. Así, el principal aspecto que lo diferencia de otros 
programas de mindfulness, como el MBSR, es la duración de las prácticas diarias formales, que en vez de ser de 45 minutos son de 15-20 minutos. Por otra parte se pone un gran énfasis en las prácticas informales, especialmente en la conciencia de la respiración y la observación de las sensaciones corporales, a la vez que se profundiza en temas de regulación emocional, relacional, comunicación consciente y se promueve el cultivo de un bienestar eudamónico.

El programa M-PBI incluye nueve módulos, ocho de dos horas cada uno (en el manual se ha añadido media hora más por sesión para las próximas implementaciones) y un retiro (prácticas intensivas) de cuatro horas dedicado exclusivamente a la realización de prácticas de mindfulness-véase Arredondo, Hurtado, Sabaté, Uriarte y Botella (2016).

El objetivo de este estudio es estimar los primeros resultados obtenidos tras la implementación del programa M-PBI, con la finalidad de valorar el aumento en la capacidad de mindfulness (mediante el cuestionario FFMQ) y de descentramiento (mediante el cuestionario EQ-D), y la disminución de síntomas de depresión, ansiedad y estrés (medida mediante la escala DASS-21).

\section{Método}

\section{Participantes}

Un total de 74 participantes (cinco grupos de M-PBI) procedentes del Institut Mensalus y del Col-legi Oficial de Psicòlegs de Catalunya participaron en el programa M-PBI para su evaluación. Todos los participantes dieron su consentimiento por escrito para participar en el estudio.

El programa de entrenamiento en mindfulness M-PBI consiste en un proceso de aprendizaje experiencial progresivo que al inicio promueve la práctica de la focalización de la atención en un objeto particular (como puede ser la respiración o las sensaciones corporales), con la finalidad de aumentar la capacidad de la mente de estar en el momento presente sin reactividad. El proceso finaliza con prácticas de monitoreo abierto con una atención receptiva tanto a la actividad interior, como a la actividad exterior, lo que promueve la práctica de mindfulness en cualquier tipo de actividad.

El programa está diseñado como un entrenamiento progresivo de ocho semanas que consta de ocho sesiones (distribuidas en diferentes módulos) de dos horas cada una, y un retiro de cuatro horas, dedicado exclusivamente a la realización de prácticas de mindfulness.

En los diversos módulos se van desarrollando aspectos esenciales de focalización de la atención, funcionamiento en modo ser, inteligencia emocional y relacional, al tiempo que se van sentando las bases de una conciencia plena y comunicación consciente-véase Arredondo et al., (2016). 


\section{Material y Procedimiento}

Cuestionario FFMQ. El Five Facet Mindfulness Questionnaire (FFMQ) es un instrumento de auto-evaluación creado por Baer et al. (Baer, Smith, Hopkins, Krietemeyer, \& Toney, 2006) que ha sido validado al español por Cebolla et al. (Cebolla et al., 2012). El cuestionario mide la tendencia general a proceder con conciencia plena en el día a día. Consta de 39 ítems que evalúan cinco facetas o factores del mindfulness: observación (ítems 1, 6, 11, 15, 20, 26, 31 у 36), descripción (ítems 2, 7, 12, 16, 22, 27, 32,37), actuar con conciencia (ítems 5, 8, $13,18,23,28,34,38$ ), ausencia de juicio (ítems $3,10,14,17,25,30,35,39$ ) y ausencia de reactividad (ítems 4, 9, 19, 21, 24, 29, 33).

Las respuestas se evalúan mediante una escala de tipo Likert de cinco puntos que evalúa la frecuencia de presentación de conductas asociadas a cada una de las facetas (de $1=$ 'nunca o muy raras veces' a $5=$ 'muy frecuentemente o siempre'). La puntuación de la escala general se obtiene sumando el valor de todos los ítems, teniendo en cuenta que los ítems $3,5,8,10,12,13,14,16,17,18,22,25,28,30$, $34,35,38$ y 39 se puntúan de forma inversa.

Escala DASS-21. La escala DASS-21 (Depression Anxiety and Stress Scale) es una versión reducida de la escala DASS-42 (Antony, Bieling, Cox, Enns, \& Swinson, 1998). Está compuesta por 21 ítems que evalúan los estados emocionales negativos de la depresión, la ansiedad y el estrés durante la última semana. Consta de 21 ítems y cada subescala tiene siete ítems. La subescala de depresión: 3, 5, 10, 13, 16, 17, 21, la de ansiedad: 2, 4, 7, 9, 15, 19, 20 y la de estrés: 1, 6, 8, 11, 12, 14, 18. Los ítems puntúan en una escala Likert de 4 categorías (desde 0 «no se aplica en nada a mí» hasta 3 «se aplica mucho a mí, o la mayor parte del tiempo»).

Para obtener la puntuación de cada subescala se suman los puntos de los ítems que las componen y por último, para obtener la puntuación total de la escala se suman los puntos de cada subescala.

Según la puntuación de cada una de las sub-escalas se establece el grado de severidad, así a mayor puntuación, mayor severidad.

Cuestionario EQ-D. El Experiences Questionnaire-Decentering (EQ-D) es una subescala de autoevaluación derivada del Experiences Questionnaire (escala de autoevaluación que originalmente media el descentramiento y la rumiación). La EQ-D fue validada por Fresco et al. (2007), y diseñada para medir la capacidad de descentramiento. La escala fue validada al español por García-Campayo et al. (2014). El descentramiento se define como la capacidad de observar los sentimientos y pensamientos como eventos temporales, es decir, es un proceso a través del cual un individuo puede distanciarse de su experiencia inmediata y observarla como un fenómeno temporal de la mente.

La subescala EQ-D está constituida por 11 ítems que evalúan tres aspectos del descentramiento: la capacidad de no verse a uno como sinónimo de sus pensamientos, la habilidad de no reaccionar a las experiencias negativas propias y la capacidad para la autocompasión. Cada ítem se puntúa con una escala tipo Likert de cinco 
puntos, por lo que la puntuación total puede variar de 11 a 55, indicando a mayor puntuación un mayor descentramiento.

Para el análisis descriptivo de las variables sociodemográficas, las puntuaciones de los distintos ítems y de las diferencias pre-post, se ha usado la media, la desviación estándar, mediana y/o rango (mínimo y máximo) para las variables numéricas y las frecuencias absolutas y relativas (\%) para las variables categóricas.

La representación gráfica de los diferencias pre-post (variable Cambio), se ha realizado mediante diagramas de caja (Boxplots), que representan el mínimo, el máximo y los tres cuartiles de la distribución empírica de una variable numérica.

El estudio de los cambios en las puntuaciones después del programa de Mindfulness se ha llevado a cabo ajustando modelos de regresión lineal incluyendo la variable Grupos de M-PBI como efecto aleatorio.

Todo el análisis estadístico se ha realizado con el paquete de software estadístico R (The R Foundation for Statistical Computing), versión 3.2.1. El nivel de significación estadística usada ha sido 0,05 .

\section{Resultados}

Del total de 74 participantes la totalidad de ellos finalizaron el estudio. La media de edad fue de 38.9 años $(\mathrm{DE}=9)$, principalmente eran de sexo femenino (74.3\%), y estaban en activo (87\%). La mayoría presentaban estudios universitarios incluyendo doctorado (94\%), y en la mitad de los casos la profesión estaba relacionada con el área de la salud. Una cuarta parte de los participantes estaban en tratamiento psicológico y la mayoría (78.6\%) no habían practicado previamente la meditación. Los motivos principales para la participación en el programa de mindfulness fueron la disminución de los síntomas, la regulación emocional y el aumento de concentración (43.8\%), el uso personal y/o profesional (32.8\%). Las características sociodemográficas y motivos de la participación en el programa se muestran en la Tabla 1.

\section{Tabla 1.}

Características sociodemográficas de la población de estudio

\begin{tabular}{lrr}
\hline Variables sociodemográficas & $\mathbf{n}$ & $(\%)^{1}$ \\
\hline Edad (años) media (DE) & 38,9 & $(9)$ \\
Sexo (mujeres), $\mathrm{n}(\%)$ & 55 & $(74.3)$ \\
Situación laboral, $\mathrm{n}(\%)$ & & \\
$\quad$ En activo & 60 & $(87)$ \\
$\quad$ De baja & 2 & $(2.9)$ \\
$\quad$ Estudiante & 7 & $(10.1)$ \\
Nivel de estudios, $\mathrm{n}(\%)$ & & \\
$\quad$ Doctorado & 8 & $(11.9)$ \\
$\quad$ Universitarios & 55 & $(82.1)$ \\
$\quad$ Secundarios & 4 & $(6)$
\end{tabular}


Profesión:

Área de la salud*

Profesiones técnicas

Estudiantes

Otras profesiones

ratamiento psicológico durante el estudio, $\mathrm{n}(\%)$

Práctica de meditación, $\mathrm{n}(\%)$

Tiempo de meditación, n (\%)

$<1$ año

1-3 años

3-5 años

$>5$ años

Motivación, n (\%)

1- Uso profesional/personal

2- Autoconocimiento/desarrollo personal/estar en el presente

3- Disminución síntomas/regulación emocional/ aumentar concentración 28

Nota. ${ }^{1}$ Los porcentajes se han calculado en el total de los datos válidos para cada variable.

Datos perdidos (n): situación laboral (5), nivel de estudios (7), profesión (5), tratamiento psicológico durante el estudio (6), práctica de meditación (4), motivación (10).

*Área de la salud: psicólogos (30); osteópata (1); médico (1); matrona (1); fisioterapeuta (2); farmacéutico(1); enfermero (1).

La puntuación media de los cuestionarios y escalas FFMQ y ED-Q aumentó de forma significativa y la puntuación media del cuestionario DASS-21 disminuyó también significativamente en la medición post-entrenamiento vs. pre-entrenamiento ( $\mathrm{p}<.0001$, en todos los casos). Los cambios más grandes se produjeron en el aumento de la capacidad de descentramiento (cambio medio de 7.6 puntos; IC95\%: 5.8 a 9.4), disminución del estrés (cambio medio de -6.6 puntos; IC95\%: -8.5 a -4.8 ), aumento del no juicio, de no reactividad y de la capacidad de observación con cambios medios de 5.8 (IC95\%: 4 a 8.1), 4.9 (IC95\%: 3.5 a 6.5) y 5.3 (IC95\%: 4.4 a 6.3) puntos, respectivamente. Por otro lado, los cambios medios fueron menores en la disminución en la ansiedad (-2.9 puntos; IC95\%: -4.8 a -1.3) y en la capacidad de describir (2.8 puntos; IC95\%: 1.8 a 3.8). En la Tabla 2 se muestran las puntuaciones medias de las distintas mediciones realizadas, el cambio observado, así como también los IC95\% para los cambios medios. En las Figuras 1,2 y 3 se representan los valores pre y post-entrenamiento de FFQM, DASS-21 y EQ-D, respectivamente. 


\section{Tabla 2}

Puntuaciones medias de los distintos cuestionarios pre- y post-entrenamiento

\begin{tabular}{llllll}
\hline Variables de medida & $\begin{array}{l}\text { Pre-ent., } \\
\text { media } \\
\text { (DE) }\end{array}$ & $\begin{array}{l}\text { Post- ent., } \\
\text { media } \\
\text { (DE) }\end{array}$ & $\begin{array}{l}\text { Cambio, } \\
\text { media (IC95\%) mediana } \\
\text { (rango) }\end{array}$ & p-valor \\
\hline FFMQ & & & & & \\
$\quad$ Observar & $23.5(6.1)$ & $28.8(4.9)$ & $5.3(4.4$ a 6.3$)$ & $5(-5,16)$ & $<.0001$ \\
$\quad$ Describir & $27.2(5.8)$ & $30(5.4)$ & $2.8(1.8$ a 3.8$)$ & $3(-9,12)$ & $<.0001$ \\
$\quad$ Actuar con & & & & & \\
conciencia & $23.2(6.8)$ & $26.9(5)$ & $3.7(2.4$ a 5.1$)$ & $4(-9,19)$ & $<.0001$ \\
$\quad$ No hacer juicios & $26.5(7.7)$ & $32.3(5.6)$ & $5.8(4$ a 8.1$)$ & $5(-7,19)$ & $<.0001$ \\
$\quad$ No reactividad & $19.2(4.1)$ & $24.1(4.1)$ & $4.9(3.5$ a 6.5$)$ & $5(-4,19)$ & $<.0001$ \\
DASS-21 & & & & & \\
$\quad$ Depresión & $10.5(8.5)$ & $4.9(5.3)$ & $-5.6(-8$ a -3.5$)$ & $-4(-28,6)$ & $<.0001$ \\
$\quad$ Ansiedad & $7.9(6.9)$ & $5(5.6)$ & $-2.9(-4.8$ a -1.3$)$ & $-2(-22,14)$ & $<.0001$ \\
$\quad$ Estrés & $17.5(8.2)$ & $10.9(6.1)$ & $-6.6(-8.5$ a -4.8) & $-6(-24,6)$ & $<.0001$ \\
EQ-D & $33.1(6.3)$ & $40.7(5.3)$ & $7.6(5.8$ a 9.4$)$ & $7(-2,20)$ & $<.0001$ \\
\hline
\end{tabular}

Nota. DASS-21, escala de depresión, ansiedad y estrés; DE, desviación estándar; EQD, cuestionario de descentramiento; FFMQ, Five Facet Mindfulness Questionnaire.
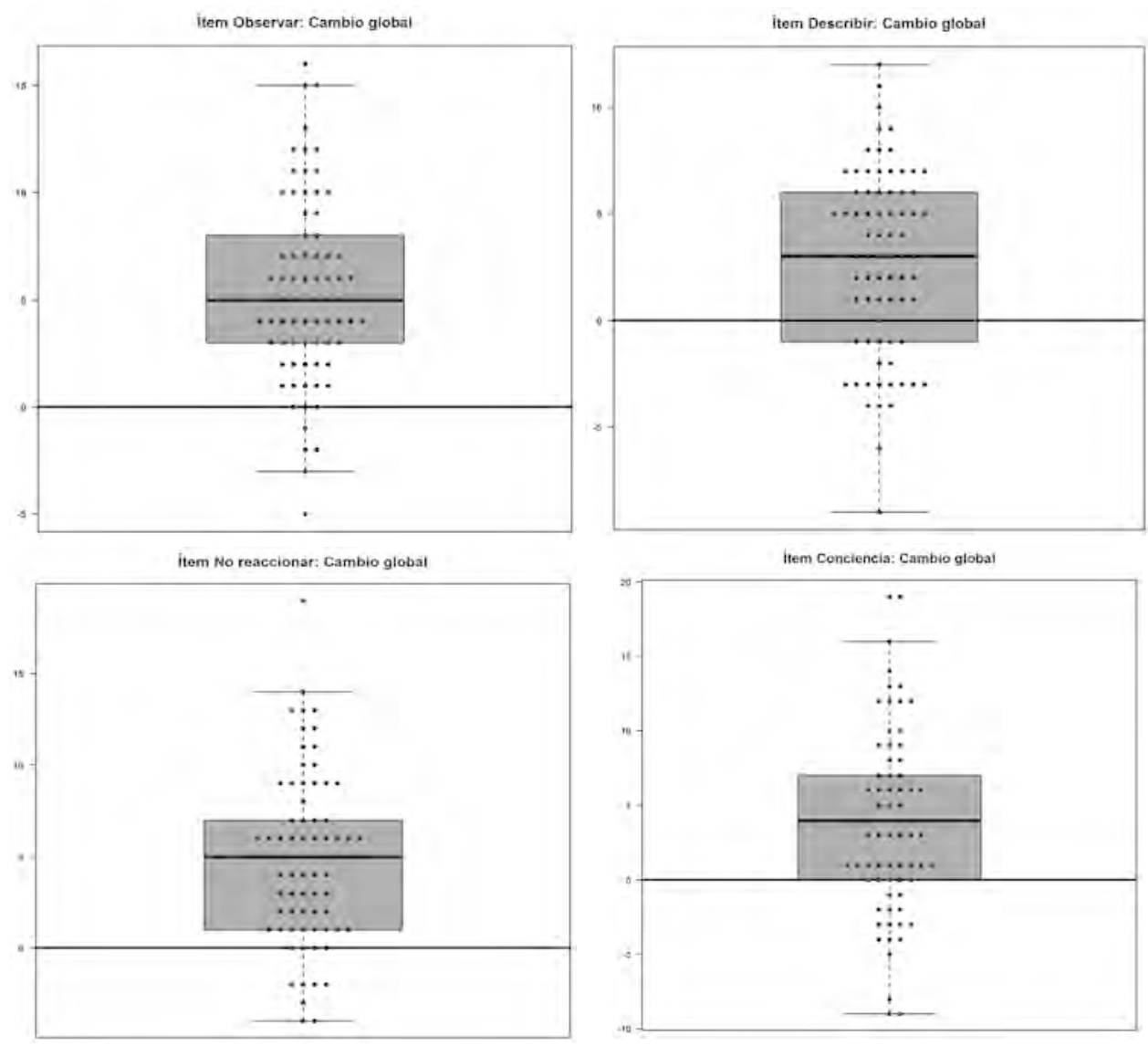


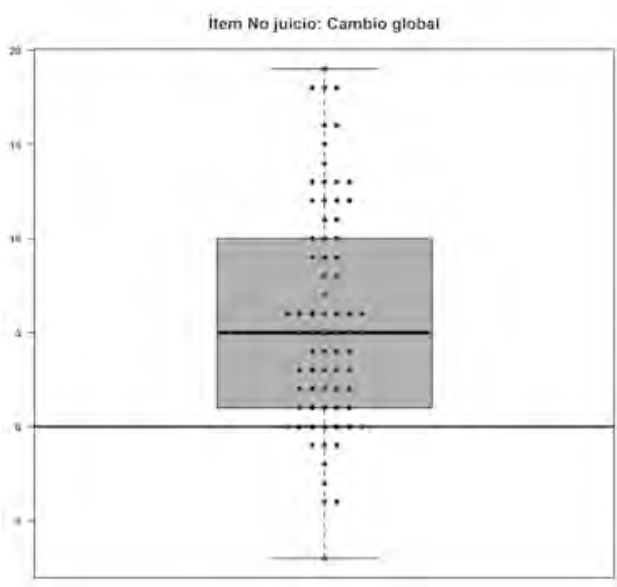

Figura 1.

Puntuaciones de las cinco facetas de FFMQ (observar, describir, no-juicio, no-reaccionar, conciencia)
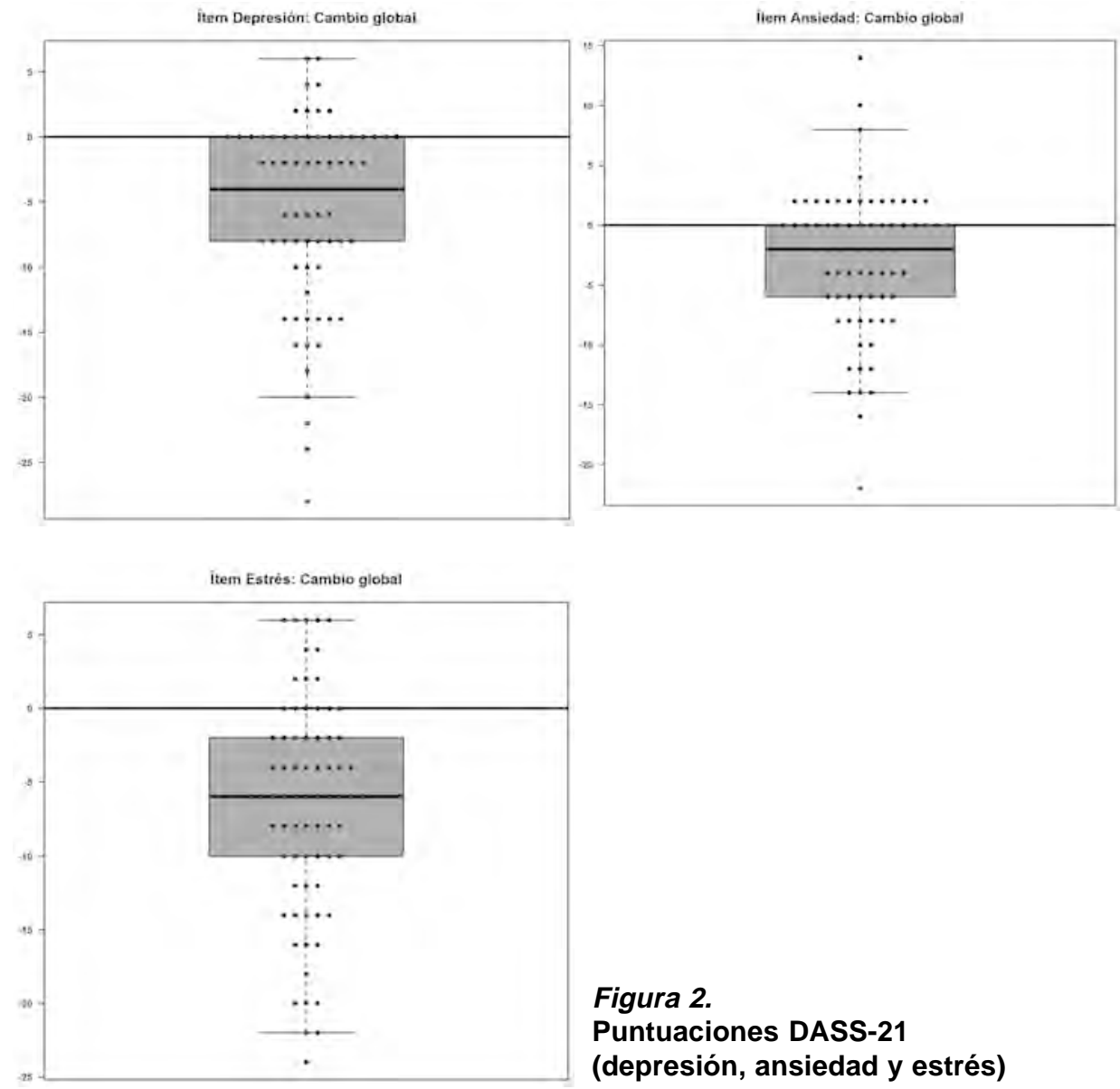

Figura 2.

Puntuaciones DASS-21

(depresión, ansiedad y estrés) 


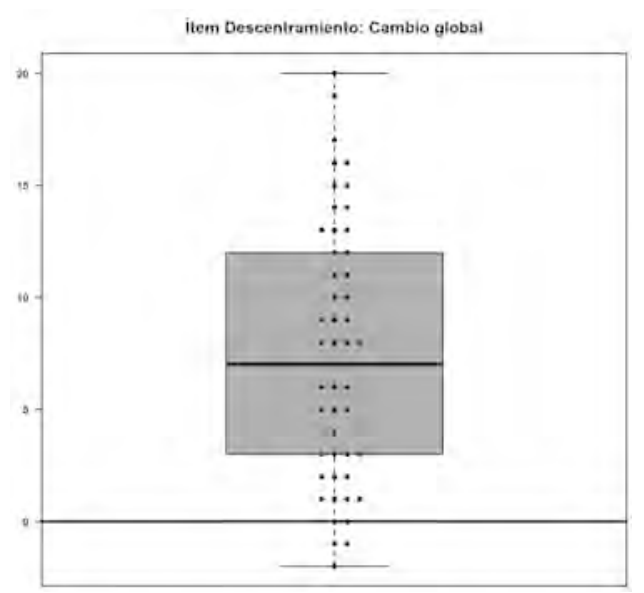

Figura 3.

Puntuaciones EQ-D

Se clasificaron los participantes según la profesión (psicólogos vs. no), hábito de meditación (sí vs. no), sexo, tratamiento psicológico (sí vs. no), y los motivos de realización del programa. Los resultados según esta clasificación mostraron que en general los participantes que eran los psicólogos, los que tenían práctica previa de meditación y los que no estaban en tratamiento psicológico presentan basalmente unas puntuaciones más altas en el FFMQ y en EQ-D y más bajas en el DASS. En relación con el sexo, se observó que los hombres tenían basalmente una puntuación más alta en el FFMQ, más baja en el EQ-D, y en el DASS-21 presentaban una puntuación más alta en la depresión y más baja en la ansiedad y estrés comparado con las mujeres. Finalmente, los participantes cuya motivación para realizar el programa fue la disminución de síntomas, regulación emocional y aumentar la concentración fueron los que presentaron puntuaciones más bajas de FFQM y EQD y más altas de DASS- 21.

Los valores medios de las diferentes mediciones pre y post-entrenamiento según profesión, práctica de meditación, sexo, tratamiento psicológico y motivación se muestran en la Tabla 3. 
Tabla 3

Puntuaciones medias de los distintos cuestionarios pre- y post-entrenamiento según profesión, práctica de meditación, sexo, tratamiento psicológico y motivación

\begin{tabular}{|c|c|c|c|c|c|c|c|c|c|c|}
\hline \multirow[t]{2}{*}{ Media (DE) } & \multicolumn{6}{|c|}{ FFMQ } & \multicolumn{3}{|c|}{$\begin{array}{c}\text { DASS- } \\
21\end{array}$} & \multirow{2}{*}{ 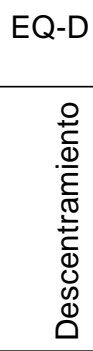 } \\
\hline & & $\begin{array}{l}\bar{\pi} \\
\geq \\
\Phi \\
\infty \\
0 \\
0\end{array}$ & 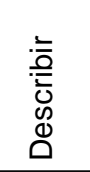 & $\frac{.0}{\frac{0}{0}}$ & 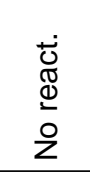 & $\begin{array}{l}\frac{\pi}{0} \\
\frac{C}{0} \\
\frac{N}{0} \\
\frac{C}{0} \\
0\end{array}$ & $\begin{array}{l}\frac{.0}{0} \\
\Phi \\
\stackrel{0}{0} \\
0\end{array}$ & $\begin{array}{l}\bar{D} \\
\frac{7}{0} \\
\frac{\mathbb{d}}{20} \\
\frac{1}{2}\end{array}$ & 离 & \\
\hline \multicolumn{11}{|l|}{ Profesión: } \\
\hline Psicólogos & Pre & $\begin{array}{l}23.7 \\
(6.1)\end{array}$ & $\begin{array}{l}28.6 \\
(4.8)\end{array}$ & $\begin{array}{l}30.3 \\
(6.5)\end{array}$ & $\begin{array}{c}20.4 \\
(4)\end{array}$ & $\begin{array}{l}24.9 \\
(5.2)\end{array}$ & $\begin{array}{l}8.5 \\
(7.9)\end{array}$ & $\begin{array}{c}5.8 \\
(5.9)\end{array}$ & $\begin{array}{l}15 \\
(7)\end{array}$ & $\begin{array}{l}34.7 \\
(5.3)\end{array}$ \\
\hline & Post & $\begin{array}{c}29 \\
(5.3)\end{array}$ & $\begin{array}{l}31.6 \\
(5.2)\end{array}$ & $\begin{array}{l}33.9 \\
(4.9)\end{array}$ & $\begin{array}{l}24.9 \\
(3.7)\end{array}$ & $\begin{array}{l}27.8 \\
(4.7)\end{array}$ & $\begin{array}{c}3.3 \\
(3.4)\end{array}$ & $\begin{array}{c}3.2 \\
(4.2)\end{array}$ & $\begin{array}{c}8.7 \\
(4.7)\end{array}$ & $\begin{array}{l}41.7 \\
(5.2)\end{array}$ \\
\hline No psicólogos & Pre & $\begin{array}{l}23.3 \\
(6.2)\end{array}$ & $\begin{array}{l}25.9 \\
(6.5)\end{array}$ & $\begin{array}{l}24.5 \\
(7.4)\end{array}$ & $\begin{array}{l}18.4 \\
(4.1)\end{array}$ & $\begin{array}{l}22.6 \\
(7.2)\end{array}$ & $\begin{array}{c}12.2 \\
(9)\end{array}$ & $\begin{array}{c}8.9 \\
(7.2)\end{array}$ & $\begin{array}{l}19.1 \\
(8.7)\end{array}$ & $\begin{array}{l}32.4 \\
(6.9)\end{array}$ \\
\hline & Post & $\begin{array}{l}28.7 \\
(4.6)\end{array}$ & $\begin{array}{l}29 \\
(5)\end{array}$ & $\begin{array}{l}31.4 \\
(6.4)\end{array}$ & $\begin{array}{l}23.6 \\
(4.5)\end{array}$ & $\begin{array}{c}26.4 \\
(5)\end{array}$ & $\begin{array}{c}6.4 \\
(6.3)\end{array}$ & $\begin{array}{c}6.5 \\
(6.4)\end{array}$ & $\begin{array}{l}12.4 \\
(6.8)\end{array}$ & $\begin{array}{c}40 \\
(5.3)\end{array}$ \\
\hline
\end{tabular}

Práct.

meditación:

$\begin{array}{lllllllllll}\text { No } & \text { Pre } & 22.5 & 26.1 & 25.5 & 18.5 & 22.2 & 11.5 & 8 & 17.8 & 32.1\end{array}$

$\begin{array}{llllllllll}\text { meditadores } & (6.1) & (6) & (7.6) & (4) & (6.6) & (8.7) & \text { (6.8) } & \text { (8.3) } & \text { (5.9) }\end{array}$

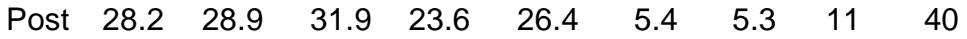

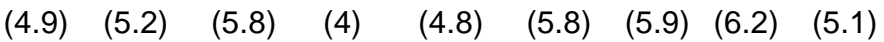

$\begin{array}{lllllllllll}\text { Meditadores } & \text { Pre } & 27 & 30.3 & 31.7 & 21.7 & 27.8 & 7.6 & 6.4 & 16.4 & 37.8\end{array}$

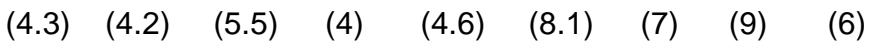

$\begin{array}{llllllllll}\text { Post } & 30.7 & 33.5 & 34.3 & 26.1 & 29.1 & 3.5 & 4 & 9.9 & 43.1\end{array}$

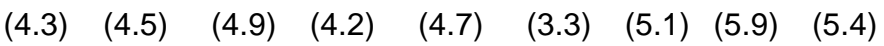

Sexo:

$\begin{array}{lllllllllll}\text { Mujeres } & \text { Pre } & 24.7 & 28.6 & 26.3 & 19.8 & 23.5 & 9.4 & 8.3 & 18 & 33.6\end{array}$

$\begin{array}{lllllllll}(5.3) & (5.1) & (7.6) & (3.7) & (5.7) & (8.2) & (7.1) & (8.1) & (6)\end{array}$

$\begin{array}{llllllllll}\text { Post } & 29.7 & 30.9 & 31.9 & 24.2 & 26.7 & 4.7 & 5.2 & 11.1 & 41.1\end{array}$

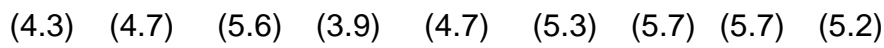

$\begin{array}{lllllllllll}\text { Hombres } & \text { Pre } & 19.8 & 23.3 & 26.9 & 17.6 & 22.4 & 13.9 & 6.7 & 16.1 & 31.9\end{array}$

$\begin{array}{lllllllll}(6.8) & (6.1) & (8.1) & (4.7) & (9.4) & (8.9) & (6.4) & (8.6) & (7.3)\end{array}$

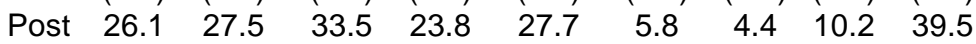

$\begin{array}{llllllll}(5.7) & (6.4) & (5.5) & (4.7) & (5.9) & (5.2) & (5.4) & (7.9)\end{array}$

Tto. psicológico:

No

$\begin{array}{llllllllll}\text { Pre } & 24 & 27.3 & 28.4 & 19.4 & 23.3 & 9.2 & 6.6 & 16.9 & 34.5\end{array}$ $\begin{array}{llllllll}(5.5) \quad(5.6) & (6.7) & (4) & (6) & (8) & (6.1) & (8.5) & (5.7)\end{array}$

$\begin{array}{llllllllll}\text { Post } & 29.3 & 30 & 33.2 & 24 & 27.4 & 4 & 4.2 & 10.3 & 41.6\end{array}$ $\begin{array}{lllllllll}(4.9) & (5.4) & (5.1) & (4.3) & (4.4) & (4.5) & (4.9) & (6.1) & (5)\end{array}$

Sí

$\begin{array}{cccccccccc}\text { Pre } & 21.5 & 25.9 & 22.2 & 18.3 & 23.5 & 15.8 & 10.9 & 20 & 30.3 \\ & (7.6) & (6.7) & (8) & (4.4) & (8.4) & (9.3) & (8.4) & (8.1) & (6.9) \\ \text { Post } & 26.8 & 29.5 & 30.3 & 24.1 & 25.7 & 8.7 & 7.5 & 12.2 & 38.4 \\ & (4.6) & (5.4) & (6.7) & (3.9) & (6.3) & (6.4) & (7.4) & (6.3) & (5.3)\end{array}$




\begin{tabular}{|c|c|c|c|c|c|c|c|c|c|c|}
\hline \multicolumn{11}{|l|}{ Motivación'1: } \\
\hline 1 & Pre & $\begin{array}{l}23.4 \\
(5.3)\end{array}$ & $\begin{array}{l}28.3 \\
(5.1)\end{array}$ & $\begin{array}{c}30 \\
(5.9)\end{array}$ & $\begin{array}{l}20.8 \\
(4.9)\end{array}$ & $\begin{array}{l}25.8 \\
(5.7)\end{array}$ & $\begin{array}{c}6.8 \\
(5.3)\end{array}$ & $\begin{array}{c}4.1 \\
(3.8)\end{array}$ & $\begin{array}{l}13.3 \\
(4.7)\end{array}$ & $\begin{array}{l}35.7 \\
(5.1)\end{array}$ \\
\hline & Post & $\begin{array}{l}28.1 \\
(5.2)\end{array}$ & $\begin{array}{c}30 \\
(5.3)\end{array}$ & $\begin{array}{l}32.8 \\
(4.5)\end{array}$ & $\begin{array}{c}24 \\
(3.3)\end{array}$ & $\begin{array}{l}27.1 \\
(4.5)\end{array}$ & $\begin{array}{c}3.1 \\
(2.9)\end{array}$ & $\begin{array}{c}3.3 \\
(3.7)\end{array}$ & $\begin{array}{c}9.4 \\
(4.3)\end{array}$ & $\begin{array}{l}41.1 \\
(4.2)\end{array}$ \\
\hline 2 & Pre & $\begin{array}{l}27.5 \\
(4.5)\end{array}$ & $\begin{array}{l}28.1 \\
(6.5)\end{array}$ & $\begin{array}{l}28.9 \\
(7.7)\end{array}$ & $\begin{array}{l}21.7 \\
(2.3)\end{array}$ & $\begin{array}{l}23.5 \\
(6.1)\end{array}$ & $\begin{array}{l}11.7 \\
(9.8)\end{array}$ & $\begin{array}{c}7.2 \\
(5.9)\end{array}$ & $\begin{array}{l}16.9 \\
(8.1)\end{array}$ & $\begin{array}{l}36.2 \\
(7.3)\end{array}$ \\
\hline & Post & $\begin{array}{l}31.1 \\
(3.9)\end{array}$ & $\begin{array}{l}31.2 \\
(5.6)\end{array}$ & $\begin{array}{l}33.6 \\
(6.1)\end{array}$ & $\begin{array}{l}25.5 \\
(4.8)\end{array}$ & $\begin{array}{c}28 \\
(4.9)\end{array}$ & $\begin{array}{c}6.1 \\
(7.6)\end{array}$ & $\begin{array}{c}4 \\
(4.4)\end{array}$ & $\begin{array}{l}11.2 \\
(7.6)\end{array}$ & $\begin{array}{c}44 \\
(6.2)\end{array}$ \\
\hline 3 & Pre & $\begin{array}{l}21.1 \\
(6.4)\end{array}$ & $\begin{array}{c}25 \\
(5.9)\end{array}$ & $\begin{array}{l}22.8 \\
(7.8)\end{array}$ & $\begin{array}{l}16.9 \\
(3.7)\end{array}$ & $\begin{array}{l}21.1 \\
(7.3)\end{array}$ & $\begin{array}{c}14.1 \\
(9)\end{array}$ & $\begin{array}{l}11.3 \\
(7.8)\end{array}$ & $\begin{array}{c}21 \\
(9.3)\end{array}$ & $\begin{array}{l}30.1 \\
(5.5)\end{array}$ \\
\hline & Post & $\begin{array}{l}27.4 \\
(4.8)\end{array}$ & $\begin{array}{l}28.8 \\
(5.5)\end{array}$ & $\begin{array}{l}31.2 \\
(6.5)\end{array}$ & $\begin{array}{l}22.9 \\
(4.3)\end{array}$ & $\begin{array}{l}25.6 \\
(5.3)\end{array}$ & $\begin{array}{c}6.4 \\
(5.4)\end{array}$ & $\begin{array}{c}7.3 \\
(7.3)\end{array}$ & $\begin{array}{l}11.7 \\
(6.9)\end{array}$ & $\begin{array}{l}38.8 \\
(5.3)\end{array}$ \\
\hline
\end{tabular}

Nota. DASS-21, escala de depresión, ansiedad y estrés; DE, desviación estándar; EQD, cuestionario de descentramiento; FFMQ, Five Facet Mindfulness Questionnaire.

${ }^{1}$ Categorías: 1. (uso profesional/personal), 2. (autoconocimiento/desarrollo personal/ estar en el presente) y 3. (disminución síntomas/regulación emocional/ aumentar concentración).

Los datos descriptivos del cambio de las diferentes mediciones realizadas (pre y post-entrenamiento) en función de la clasificación previamente mencionada se muestran en las Tablas 4 y 5 . De forma general, se observa que los participantes que no eran psicólogos versus los psicólogos presentaban un mayor cambio principalmente en el aumento del no juicio (cambio de 6 vs. 2.5 puntos). Asimismo, los que estaban en tratamiento psicológico comparado con los que no lo estaban mostraban un mayor cambio en el aumento del no juicio (cambio de 6 vs. 2 puntos) y una mayor disminución del estrés (cambio de -10 vs. -6 puntos). En el caso de los participantes que no tenían práctica de meditación previa al inicio del programa se percibió un mayor cambio en el aumento del no juicio (cambio de 6 vs. 2 puntos) y de la conciencia (cambio de 4 vs. 1 puntos), así como una mayor reducción de la ansiedad (cambio de - 4 vs. - 1 puntos). La comparación por sexos mostró como más relevante que los hombres presentaban una mayor disminución de la depresión (cambio de 7 vs. -4 puntos), seguido por un mayor aumento de la capacidad de descripción (cambio de 5 vs. 2 puntos), mientras que las mujeres tenían un mayor cambio en el aumento del descentramiento (cambio de 8 vs. 6.5 puntos). Finalmente, la comparación de la motivación para la realización del programa de mindfulness evidenció que los participantes cuyo motivo era la disminución de síntomas, regulación emocional y aumentar la concentración eran los presentaban un mayor cambio en el aumento de no juicio (cambio de 10 vs. 2 y 3 puntos en los otros dos grupos de motivación), en la reducción del estrés (cambio de -10 vs. -2 y -4 puntos) y en la disminución de la depresión (cambio de -6 vs. -2 y -4 puntos). 


\section{Tabla 4}

Cambio del FFMQ post-entrenamiento vs. pre-entrenamiento según profesión, práctica de meditación, sexo, tratamiento psicológico y motivación Mediana del cambio (rango) FFMQ

Mediana del cambio (rango) FFMQ

\begin{tabular}{llllll}
\hline & Observar & Describir & No juicio & No react. Conciencia \\
\hline Profesión: & & & & & \\
$\quad$ Psicólogos & $4(-2,15)$ & $3(-9,10)$ & $2,5(-4,19)$ & $5(-4,12)$ & $3(-5,13)$ \\
$\quad$ No psicólogos & $5(-5,15)$ & $4(-6,12)$ & $6(-7,18)$ & $5(-4,19)$ & $4(-9,19)$ \\
Práctica meditación & & & & & \\
$\quad$ Meditadores & $4(-2,9)$ & $4(-3,10)$ & $2(-3,8)$ & $5(-4,12)$ & $1(-9,8)$ \\
$\quad$ No meditadores & $5(-5,15)$ & $3(-9,12)$ & $6(-7,19)$ & $5(-4,19)$ & $4(-9,19)$ \\
Sexo: & & & & & \\
$\quad$ Hombres & $5(-5,15)$ & $5(-6,9)$ & $6(1,18)$ & $6(-4,19)$ & $4(-8,19)$ \\
$\quad$ Mujeres & $4(-3,16)$ & $2(-9,12)$ & $5(-7,19)$ & $4(-4,14)$ & $3(-9,19)$ \\
Tratamiento psicológico: & & & & & \\
$\quad$ Sí & $4(-2,15)$ & $4(-6,12)$ & $10(0,18)$ & $5(0,13)$ & $1(-8,14)$ \\
$\quad$ No & $5(-5,15)$ & $3(-9,11)$ & $4(-7,19)$ & $5(-4,19)$ & $4(-9,19)$ \\
Motivación*: & & & & & \\
$\quad 1$ & $4(0,12)$ & $2(-9,9)$ & $2(-4,15)$ & $4(-4,12)$ & $1(-9,13)$ \\
$\quad 2$ & $4(-3,10)$ & $5(-4,10)$ & $3(-1,19)$ & $5(-2,10)$ & $4(-3,14)$ \\
3 & $5,5(-5,15)$ & $4,5(-6,12)$ & $10(-7,18)$ & $6(-4,19)$ & $5,5(-8,19)$ \\
\hline
\end{tabular}

Nota. FFMQ, Five Facet Mindfulness Questionnaire.

*Motivación: 1 (uso profesional/personal), 2 (autoconocimiento/desarrollo personal/estar en el presente) y 3 (disminución síntomas/regulación emocional/ aumentar

concentración). 


\section{Tabla 5}

Cambio de DASS-21 y EQ-D post-entrenamiento vs. pre-entrenamiento según profesión, práctica de meditación, sexo, tratamiento psicológico y motivación

\begin{tabular}{lllll}
\hline \multicolumn{2}{l}{ Mediana del cambio (rango) } & DASS-21 & & EQ-D \\
\hline & Depresión & Ansiedad & Estrés & Descentramiento \\
\hline $\begin{array}{lllll}\text { Profesión: } \\
\quad \text { Psicólogos }\end{array}$ & $-3(-24,2)$ & $-1(-22,10)$ & $-6(-20,6)$ & $7(-2,19)$ \\
$\quad$ No psicólogos & $-4(-28,6)$ & $-2(-14,14)$ & $-6(-24,6)$ & $7(-1,20)$ \\
Práctica de meditación: & & & & \\
$\quad$ Meditadores & $-4(-28,6)$ & $-1(-22,14)$ & $-6(-24,6)$ & $5,5(0,12)$ \\
$\quad$ No meditadores & $-2(-14,2)$ & $-4(-14,10)$ & $-6(-22,6)$ & $7(-2,20)$ \\
Sexo: & $-7(-28,6)$ & $-1(-16,10)$ & $-7(-22,6)$ & $6,5(-2,20)$ \\
$\quad$ Hombres & $-4(-20,6)$ & $-2(-22,14)$ & $-6(-24,6)$ & $8(-1,17)$ \\
$\quad$ Mujeres & & & & \\
Tratamiento psicológico: & $-4(-28,6)$ & $-2(-22,14)$ & $-10(-22,6)$ & $6,5(-2,19)$ \\
$\quad$ Sí & $-3(-24,6)$ & $-1(-16,10)$ & $-6(-24,6)$ & $8(-2,19)$ \\
$\quad$ No & & & & \\
Motivación*: & & & \\
$\quad 1$ & $-2(-16,2)$ & $0(-8,10)$ & $-2(-16,6)$ & $6(-2,14)$ \\
$\quad 2$ & $-4(-22,4)$ & $-2(-12,2)$ & $-4(-20,6)$ & $7(0,16)$ \\
3 & $-6(-28,6)$ & $-2(-22,14)$ & $-10(-24,6)$ & $7(-1,20)$ \\
\hline
\end{tabular}

Nota. DASS-21, escala de depresión, ansiedad y estrés; EQ-D, cuestionario de descentramiento.

*Motivación: 1 (uso profesional/personal), 2 (autoconocimiento/desarrollo personal/estar en el presente) y 3 (disminución síntomas/regulación emocional/ aumentar concentración).

Los resultados de los modelos lineales mixtos para los cambios en cada una de las mediciones realizadas (FFMQ, DASS-21 y EQ-D) indican que no hay asociación estadísticamente significativa entre los cambios observados y las variables edad, sexo, el estar en tratamiento psicológico, profesión, tener práctica de meditación y la motivación para realizar el programa de entrenamiento en mindfulness.

\section{Discusión}

Los resultados de este estudio muestran que el entrenamiento en un programa de mindfulness utilizando prácticas formales breves permite aumentar la capacidad de observar, describir, actuar con conciencia, no juzgar y disminuir la reactividad, así como reducir el estrés, la ansiedad y la depresión, y aumentar la capacidad de descentramiento. Los cambios observados son similares a los descritos en otros estudios en los que se han implementado programas de mindfulness breves, aunque en algunos casos los valores no son comparables ya que utilizan otros instrumentos de medida (Bergen-Cico, Possemato, \& Cheon, 2013; Chen, Yang, Wang, \& Zhang, 2013; Gluck \& Maercker, 2011; Mackenzie, Poulin, \& Seidman-Carlson, 2006; Phang, Mukhtar, Ibrahim, Keng, \& Mohd. Sidik, 2015; Tang et al., 2007; Zeidan et al., 2010). 
La comparación de los valores basales del FFMQ con los publicados en la población española muestra valores medios similares (Cebolla et al., 2012), así como también son comparables con los valores basales de una población de profesionales de atención primaria en España que realizó el programa MBSR. En relación con este último estudio se observaron valores de cambio del FFMQ parecidos a los que se presentan en este estudio, aunque en nuestro estudio las prácticas de meditación eran breves.

Cabe resaltar que en algunos casos los cambios observados en nuestro estudio fueron ligeramente más altos (cambio medio de descentramiento 2.8 vs. 1.9, actuar con conciencia 3.7 vs. 3 , ausencia de juicio 5.8 vs. 4.1 , ausencia de reactividad 4.9 vs. 3.2), mientras que el cambio en la capacidad de observar fue menor (5.3 vs. 6.3). No obstante, hay que tener en cuenta las diferencias en las características de las poblaciones, tratándose en nuestro caso de una población más heterogénea en relación con el área profesional con un 55\% de los participantes relacionados con el área de la salud y una media de edad de 39 años, mientras que el estudio con el que se compara son todos profesionales de atención primaria con una edad media de 47 años (Martín, Rodríguez, Pujol-Ribera, Berenguera, \& Moix, 2013).

Los mayores cambios que se han observado en este estudio han sido en el aumento del descentramiento, disminución del estrés, ausencia de juicio y de reactividad, y aumento de la capacidad de observación. A pesar de que la ansiedad es el aspecto que presenta un menor cambio, este es significativo comparado con los valores previos al inicio del programa. En relación con este punto, algunos estudios han mostrado que programas de mindfulness breves producen cambios en el estado psicológico de los participantes pero no en la reducción de la ansiedad. Bergen-Cico et al. (2013) presentan los resultados de los beneficios potenciales en el estado psicológico de un programa de mindfulness breve que corresponde a una modificación del programa MBSR. Este programa tiene una duración de cinco semanas, con sesiones semanales de $2.5 \mathrm{~h}$. Los resultados muestran que los participantes experimentan cambios significativos en aspectos de mindfulness y de autocompasión. Sin embargo, no se observan cambios en la ansiedad, sugiriendo que esta es más resistente al cambio y la necesidad de programas de mindfulness con un periodo de tiempo más prolongado para poder reducirla.

En nuestro estudio, el programa de mindfulness consta de prácticas breves pero mantiene las ocho semanas de duración del programa MBSR. Los resultados obtenidos indican que la reducción del tiempo de las prácticas, manteniendo las ocho semanas de duración del programa, permite obtener cambios significativos tanto en los aspectos de mindfulness, como de descentramiento, así como en la reducción de la sintomatología, incluyendo la ansiedad. Asimismo, cabe resaltar que los participantes sin práctica previa de meditación presentaban un cambio ligeramente más importante en la reducción de la ansiedad comparado con los que ya meditaban anteriormente, aunque estos últimos alcanzaron una puntuación postentrenamiento más baja. No obstante, los cambios observados en las diferentes 
mediciones fueron independientes de la edad, el sexo, el estar en tratamiento psicológico, profesión, tener práctica de meditación y de la motivación.

Por otro lado, es fundamental destacar la importancia de las prácticas breves en el cumplimento de los participantes del programa. Algunos estudios han mostrado una tasa importante de abandonos en el grupo que realizaba el programa de mindfulness sugiriendo como causa principal la falta de tiempo por parte de los participantes (Shapiro et al., 2005). En relación con lo expuesto anteriormente, los resultados de un estudio piloto no controlado en el que se implementó una versión abreviada del programa MBSR de 8 semanas con prácticas de meditación de 10-20 minutos diarios mostró cambios significativos en la reducción de la depresión, ansiedad y estrés, así como también una mejoría en la satisfacción laboral (Fortney et al. 2013). Se observó que al final del programa únicamente un participante había abandonado el estudio. Abordar esta restricción es particularmente importante dado las limitaciones de tiempo que actualmente tiene la mayoría de la población. De aquí la importancia de valorar los resultados de programas de mindfulness con prácticas breves que permitan mantener el cumplimiento de los participantes. En nuestro estudio la totalidad de los participantes finalizó el estudio.

Este estudio presenta algunas limitaciones, como son:

(1) Los participantes en el estudio fueron personas que de forma voluntaria quisieron participar y por tanto tenían un grado de motivación que podría ser más alto que el de otras poblaciones de estudio, lo que puede limitar su validez externa.

(2) A pesar de que la mejoría de las distintas mediciones fueron independientes del sexo, la edad, el tratamiento psicológico, la profesión, la práctica previa de meditación y la motivación, no podemos excluir que otras variables puedan haber influido en la mejoría de estas mediciones, por lo que se requiere de un estudio que incluya un grupo de control que permita valorar el efecto de acontecimientos externos que puedan haber influenciado en algunas de estas mediciones.

(3) Aunque se trata de una población heterogénea en relación a la profesión, la mayoría está relacionada con el ámbito de la salud por lo que se debe ser cauteloso en la generalización de estos datos. Se requiere de una distribución más representativa de los distintos ámbitos laborables para poder hacerlo extensivo a una población general.

(4) Finalmente, otra limitación es la falta de un seguimiento después de la finalización del entrenamiento para evaluar el mantenimiento o no de los cambios alcanzados.

A modo de resumen, estos primeros resultados obtenidos del programa M-PBI permiten mostrar que un programa de mindfulness que utiliza prácticas formales breves, mejora la capacidad mindfulness y de descentramiento, a la vez que disminuye la sintomatología de depresión, ansiedad y estrés. No obstante, se requiere de futuros estudios que presenten un diseño aleatorizado, con grupo control para confirmar estos resultados y poder controlar los posibles factores confusores y minimizar los sesgos. En este sentido, ya se ha realizado un ensayo 
clínico, aleatorizado y controlado en el ámbito de una empresa privada con el objetivo de valorar la eficacia de este programa en la reducción del estrés laboral, la capacidad mindfulness, el descentramiento y la disminución del burnout tras ocho semanas de la participación en el programa, y con un seguimiento a los tres meses. Los resultados de este estudio serán publicados en breve.

\section{Agradecimientos:}

Agradecemos especialmente a los participantes del programa por su extraordinaria contribución. Al Institut Mensalus de Barcelona, al Col•legi Oficial de Psicòlegs de Catalunya y a la Facultat de Psicologia, Ciències de l'Educació i l'Esport (FPCEE) Blanquerna de la Universitat Ramon Llull su colaboración en la implementación del programa M-PBI. A The Compassionate Mind Fundation y Paul Gilbert por compartir generosamente sus investigaciones y teoría. Al Equipo de Investigación de Psicología del deporte de la Universitat Autònoma de Barcelona, Lluis Capdevila y Jordi Moreno. A Ausiàs Cebolla, Javier García Campayo, Joaquim Soler Albert Feliu, Marta Alonso, Mayte Miró y Vicente Simón por su orientación y ser fuente de inspiración.

\section{Referencias bibliográficas}

Antony, M. M., Bieling, P. J., Cox, B. J., Enns, M. W., \& Swinson, R. P. (1998). Psychometric properties of the 42-item and 21-item versions of the Depression Anxiety Stress Scales in clinical groups and a community sample. Psychological Assessment, 10(2), 176-181. doi:http://doi.org/10.1037/1040-3590.10.2.176

Arredondo, M., Hurtado, P., Sabaté, M., Uriarte, C. y Botella, L. (2016). Programa de Entrenamiento en Mindfulness Basado en Prácticas Breves Integradas (M-PBI). Revista de Psicoterapia, (27)103, 133-150. http://revistadepsicoterapia.com/rp103-10.html

Baer, R. A., Smith, G. T., Hopkins, J., Krietemeyer, J., \& Toney, L. (2006). Using self-report assessment methods to explore facets of mindfulness. Assessment, 13(1), 27-45. doi: http://doi.org/10.1177/1073191105283504

Bergen-Cico, D., Possemato, K., \& Cheon, S. (2013). Examining the efficacy of a brief mindfulness-based stress reduction(Brief MBSR) program on psychological health.Journal of American College Health, 61(6), 348360. doi:http://doi.org/10.1080/07448481.2013.813853

Bishop, S. R. (2002). What do we really know about mindfulness-based stress reduction? Psychosomatic Medicine, 64(1), 71-83. Retrieved from http://www.ncbi.nlm.nih.gov/pubmed/11818588

Cebolla, A., García-Palacios, A., Soler, J., Guillen, V., Baños, R., \& Botella, C. (2012). Psychometric properties of the Spanish validation of the Five Facets of Mindfulness Questionnaire (FFMQ). The European Journal of Psychiatry. http://doi.org/10.4321/S0213-61632012000200005

Chen, Y., Yang, X., Wang, L., \& Zhang, X. (2013). A randomized controlled trial of the effects of brief mindfulness meditation on anxiety symptoms and systolic blood pressure in Chinese nursing students. Nurse Education Today, 33(10), 1166-1172. doi:http://doi.org/10.1016/j.nedt.2012.11.014

Fortney, L., Luchterhand, C., Zakletskaia, L., Zgierska, A., \& Rakel, D. Abbreviated mindfulness intervention for job satisfaction, quality of life, and compassion in primary care clinicians: a pilot study. Annals of Family Medicine, 11(5), 412-420. doi:http://doi.org/10.1370/afm.1511

Fresco, D. M., Moore, M. T., van Dulmen, M. H. M., Segal, Z. V, Ma, S. H., Teasdale, J. D., \& Williams, J. M. G. (2007). Initial psychometric properties of the experiences questionnaire: validation of a self-report measure of decentering. Behavior Therapy, 38(3), 234-246. doi:http://doi.org/10.1016/j.beth.2006.08.003 
Garcia-Campayo, J., Navarro-Gil, M., Andrés, E., Montero-Marin, J., López-Artal, L., \& Demarzo, M. M. P. (2014). Validation of the Spanish versions of the long (26 items) and short (12 items) forms of the SelfCompassion Scale (SCS). Health and Quality of Life Outcomes, 12, 4. doi:http://doi.org/10.1186/14777525-12-4

Gluck, T. M., \& Maercker, A. (2011). A randomized controlled pilot study of a brief web-based mindfulness training. BMC Psychiatry, 11, 175. doi:http://doi.org/10.1186/1471-244X-11-175

Instituto esMindfulness. (n.d.). Entrenando Mindfulness con MBSR. Http://www.esmindfulness.com (con Acceso El 14 de Febrero).

Kabat-Zinn, J. (1994). Wherever you go, there you are: mindfulness meditation in everyday life. Hyperion.

Mackenzie, C. S., Poulin, P. A., \& Seidman-Carlson, R. (2006). A brief mindfulness-based stress reduction intervention for nurses and nurse aides. Applied Nursing Research, 19(2), 105-109. doi:http://doi.org/ 10.1016/j.apnr.2005.08.002

Martín Asuero, A., Rodríguez Blanco, T., Pujol-Ribera, E., Berenguera, A., \& Moix Queraltó, J. (2013). Evaluación de la efectividad de un programa de mindfulness en profesionales de atención primaria. Gaceta Sanitaria, 27(6), 521-528. doi:http://doi.org/10.1016/j.gaceta.2013.04.007

Pérez, M. A., y Botella, L. (2006). Conciencia plena (mindfulness) y psicoterapia: Concepto, evaluación y aplicaciones clínicas. Revista de Psicoterapia, 66/67, 77-120. http://revistadepsicoterapia.com/concienciaplena-mindfulness-y-psicoterapia-concepto-evaluacion-y-aplicaciones-clinicas.html

Phang, C. K., Mukhtar, F., Ibrahim, N., Keng, S.-L., \& Mohd. Sidik, S. (2015). Effects of a brief mindfulness-based intervention program for stress management among medical students: the Mindful-Gym randomized controlled study. Advances in Health Sciences Education, 20(5), 1115-1134. doi:http://doi.org/10.1007/ s10459-015-9591-3

Shapiro, S. L., Astin, J. A., Bishop, S. R., \& Cordova, M. (2005). Mindfulness-based stress reduction for health care professionals: Results from a randomized trial. International Journal of Stress Management, 12(2), 164176. doi:http://doi.org/10.1037/1072-5245.12.2.164

Simón, V. (2007). Mindfulness y neurobiología. Revista de Psicoterapia, 17(66/67), 5-30. http:// revistadepsicoterapia.com/mindfulness-y-neurobiologia.html

Tang, Y.-Y., Ma, Y., Wang, J., Fan, Y., Feng, S., Lu, Q., ... Posner, M. I. (2007). Short-term meditation training improves attention and self-regulation. Proceedings of the National Academy of Sciences of the United States of America, 104(43), 17152-17156. doi:http://doi.org/10.1073/pnas.0707678104

Zeidan, F., Johnson, S. K., Diamond, B. J., David, Z., \& Goolkasian, P. (2010). Mindfulness meditation improves cognition: evidence of brief mental training. Consciousness and Cognition, 19(2), 597-605. doi:http:// doi.org/10.1016/j.concog.2010.03.014 